\title{
Germanica
}

\section{Georg Simmel : métaphysique du laconisme}

Georg Simmel

\section{Bertrand Saint-Sernin}

\section{(2) OpenEdition}

Journals

Édition électronique

URL : http://journals.openedition.org/germanica/2431

DOI : 10.4000/germanica.2431

ISSN : 2107-0784

Éditeur

Université de Lille

\section{Édition imprimée}

Date de publication : 31 décembre 1990

Pagination : 53-65

ISBN : 9782913857025

ISSN : 0984-2632

\section{Référence électronique}

Bertrand Saint-Sernin, « Georg Simmel : métaphysique du laconisme », Germanica [En ligne], 8| 1990, mis en ligne le 25 novembre 2014, consulté le 06 octobre 2020. URL : http://journals.openedition.org/ germanica/2431 ; DOI : https://doi.org/10.4000/germanica.2431

Ce document a été généré automatiquement le 6 octobre 2020.

(C) Tous droits réservés 


\title{
Georg Simmel : métaphysique du laconisme $^{1}$
}

Georg Simmel

\author{
Bertrand Saint-Sernin
}

1 Simmel n'est pas un auteur facile : il est limpide, certes, mais préfère à la lumière crue du concept l'éclairage latéral de l'esquisse ; il décrit, dans la réalité sociale ou dans l'existence des individus, des dispositions ou des attitudes, dont le principe générateur et le sens ne restent visibles qu'un court instant. Non qu'il s'attache, tel un esthète, au seul éphémère: il redoute seulement que le sable de l'habitude ne recouvre et ne masque le moment décisif, le fait révélateur. Comme il ne dédaigne pas de réfléchir sur des objets en apparence mineurs (la mode, les ruines, les paysages, l'aventure, le visage, le comédien, les aphorismes, etc.), on a cru qu'il n'avait de la société qu'une vision fragmentée. Il en va tout autrement: Simmel pense que les événements universels ne touchent les individus qu'en modifiant leur vie proche. Il tente de relier le cours de l'histoire aux détails des existences. Aussi son œuvre comporte-t-elle des essais et des traités : dans les uns, campé sur la proue pour une navigation exploratoire, il lance hardiment sa sonde; dans les autres, systématique, il dresse des cartes. Ces deux opérations s'inspirent, on le devine, de la même intention: mettre en relief puis en rapport les deux registres, distincts et indissociables, de la causalité et du sens. Ses travaux sur l'argent, son épistémologie de l'histoire, ses leçons sur de grands philosophes témoignent de l'ampleur de son champ de vision. En même temps, il réalise des percées plutôt qu'il n'établit durablement un système. Il reste philosophe, épris d'idées dérangeantes, et par là éclairantes.

2 L'analyse sociale, la perspicacité historique procèdent non seulement du savoir, mais du jugement : entre l'intuition ordinaire et la compréhension historique, la différence n'est pas de nature. La science historique s'édifie sur le terreau de l'expérience, synthèse de deux éléments: d'un côté, des informations factuelles, dérivant de perceptions directes ou de témoignages; de l'autre, l'attribution à des agents humains, comme à leur source unique et continue, des péripéties ainsi relatées. L'idéal d'un Ranke, assignant à l'Histoire de restituer «ce qui s'est réellement passé » est 
inaccessible et illusoire; la tâche de l'historien consiste à mettre en relief, dans la profusion en droit indéfinie des événements, des actions entremêlées tissant le cours intelligible des choses. Ce parti pris kantien accrédite à première vue le relativisme supposé de l'auteur: toute œuvre historique apparait comme un système de représentations, dont seules les qualités intellectuelles et éthiques de leur créateur garantissent la valeur. Les raisons de Simmel pour réfuter l'ontologie réaliste de Ranke, en matière d'histoire, sont pourtant d'un autre ordre : il ne doute aucunement que le concept de vérité, au sens traditionnel et encore kantien d'adaequatio rei et intellectus, s'applique aux événements rapportés par l'historien: il pense que les choses qui ont effectivement eu lieu se sont produites d'une manière singulière, unique, et qu'elles constitueraient bien la matière de l'histoire, si elles étaient susceptibles d'être remémorées et revécues. Le paradoxe est le suivant : «Je suis persuadé, dit Simmel, que nous percevons l'homme tout entier» (Das Individum und die Freiheit, p. 65). Mais cette perception s'effectue sur la base de données fragmentaires. Nous nommons « âme » le corrélat réel du principe de continuité spirituelle que nous prêtons à tout existant. Or, justement, l'histoire n'est pas une résurrection intégrale du passé : nous faisons subir aux événements et aux agents des modifications caractéristiques, qui rendent possible, à partir de représentations partielles, une reconstruction cohérente du passé en tant qu'histoire. La compréhension historique n'est qu'une modification de l'intelligence que nous avons de ce qui se passe actuellement : « en fin de compte, dans la mesure où ils sont convenablement peints, le portrait et les actions d'un personnage historique sont devant nous comme le portrait et les actions d'une personne que nous connaissons " (I.u.F., p. 65). Dans cette compréhension de l'Autre, interfèrent des données singulières et des éléments intemporels.

3 Simmel n'a aucune tentation solipsiste: l'aperception de l'Autre est originaire; la polarité entre le «tu » et le « je » est inscrite dans notre être. Bien plus, nous l'avons dit, nous percevons l'homme tout entier. Cette perception de l'existence totale de l'autre, même confuse et fragmentaire, même éveillée par des incidents particuliers, «n'en demeure pas moins la manière fondamentalement une selon laquelle l'homme agit sur l'homme » (I.u.F., p. 65).

4 Illustrant ses hypothèses par l'analyse d'œuvres d'art comme le Faust de Goethe, il montre notamment que l'acte de comprendre va de l'idéal au réel et du typique au concret: tout chef-d'œuvre, outre le sens que son auteur lui a donné, comporte une pluralité de significations virtuelles que ses lecteurs, au fil du temps, lui confèrent ; ces sens, auxquels l'artiste n'avait pas songé, n'en sont pas moins admissibles, dès qu'ils jettent sur une action une lumière cohérente. Devant les archives du passé, l'observateur contemporain, historien ou simple lecteur, se trouve dans une position analogue : il n'y a bien qu'une seule vérité historique, un seul cours effectif des choses ; mais, par leur nature même, les relations d'événements passés subissent d'inévitables inflexions, s'adaptent à divers moules, ne nous touchent que si, d'une certaine façon, nous les intégrons à notre expérience possible. Et réciproquement, œuvres d'imagination et récits historiques nous fournissent des grilles de lecture, pour interpréter l'état présent des choses. « Une création de l'esprit [...] peut se comparer à une énigme que son inventeur a conçue pour recevoir une solution déterminée. Mais si quelqu'un trouve une seconde solution, tout aussi admissible du point de vue logique et poétique, celle-ci a autant de valeur que celle à laquelle l'artiste avait songé » (I.u.F., 
p. 73). Le même raisonnement s'applique aux systèmes techniques et à leur perfectionnement.

5 La constitution de l'histoire, chez l'historien et chez son public, est donc un processus qui ne laisse indemnes ni les acteurs ni la matière : la compréhension historique est un genre, une modalité précise, cernable par concepts, de la relation des hommes entre eux. La scène historique n'est pas le monde naturel; les agents historiques ne sont pas exactement des hommes comme nous, comme ceux que nous rencontrons dans la vie de tous les jours; le cours de l'histoire n'est pas non plus identique à la réalité des existences entremêlées, dans leur profusion et leur obscurité. "L'histoire n'est pas le passé qui, pris comme il se donne immédiatement à nous, est fait de fragments discontinus. C'est une forme, ou une somme de formes déterminées, grâce auxquelles l'esprit, par observation et synthèse, pénètre et maîtrise une matière antérieurement donnée, legs de ce qui est advenu. Par le fait que je saisis comme historique une suite (s.e. d'événements), je n'enrichis pas leur contenu; j'introduis ou conforte seulement un mode de liaison fonctionnel de ce qui est donné à l'intuition interne » (I.u.F., p. 71).

6 Simmel a décrit le système des modifications par lesquelles les historiens, sans cesser de se réclamer du concept aristotélicien ou sémantique de vérité, édifient des œuvres qui ne sont en aucun cas l'image ou la restitution fidèles du passé. Les concepts d'âme, de destin et d'action interviennent comme des opérateurs nécessaires pour transformer le passé en une scène historique et les hommes du passé en agents historiques. Le lien qui unira ces agents entre eux sera lui-même double : dans la mesure où ils sont insérés dans un univers de forces et de besoins, leurs interactions seront causales; dans la mesure où ces mêmes agents font des projets et où leur vie a un sens, leurs liens seront symboliques et signifiants. Simmel réfute l'objection matérialiste selon laquelle n'existent que des conditions objectives et des forces. L'ordre de la signification est réel et irréductible, dans la mesure où les individus, en tant qu'agents de l'histoire, ne sont pas assimilables à des choses. Même soumis, même réduits en servitude, ils ne sont pas réifiables. " Au sein de ce qui nous advient et qui nous ballotte, nous sentons en nousmêmes, avec plus ou moins de certitude, un télos au moins formel, une réalisation de nos dispositions, le déploiement d'un germe que nous recelons, ou que nous sommes » (I.u.F., p. 80).

7 Cette position de Simmel ne dérive pas de l'observation, elle procède d'un postulat. Plutôt que kantien, il est spinoziste. Sa conception du "destin » est à cet égard révélatrice. Reprenant un thème stoïcien bien connu, il note que toute existence peut se regarder selon une double perspective : en tant qu'elle est immergée dans l'univers, soumise à des pressions et à des forces, elle constitue un fragment du cosmos, une parcelle infime de la réalité une. Pour saisir ainsi une vie, il suffit d'observer les puissances qui agissent sur elle, les causes qui, de l'extérieur, la perturbent ou la modifient. En même temps, toutes ces péripéties sourdent de l'individu, ont en lui leur origine et l'amènent à en revendiquer la responsabilité, ne serait-ce que pour leur reconnaître un sens.

8 Simmel nomme destin (Schicksal) l'affirmation simultanée, ou plutôt la synthèse, de ces deux ordres irréductibles, celui de la causalité cosmique et de la signification libre, et il ajoute que leur rencontre confère aux événements de l'existence le double caractère de la nécessité et de la contingence. La sagesse, pour Simmel comme pour Spinoza, consiste à regarder ce qu'on vit comme s'il s'agissait d'événements extérieurs, cosmiques ; et à regarder ce qui procède de l'univers comme s'il était intérieur, parce 
que nous participons de son cours et de son ordre. Cette position est à la fois rationaliste et mystique. Elle échappe souvent aux lecteurs de Simmel. Pourtant, dans Das Problem des Schicksals (I.u.F., p. 17), Simmel est clair : «Qu'il subsiste en tout ce que nous nommons notre destin, qu'il soit favorable ou destructeur, un élément qui non seulement échappe à notre entendement, mais qui, tout en faisant partie du projet de notre vie, ne soit pourtant pas entièrement assimilé, cela s'accorde, conformément à la structure globale du destin, à ce sentiment étrange selon lequel le nécessaire dans notre vie est aussi en quelque sorte contingent $»$.

«Tenir sous le destin », «Unter dem Schicksal stehen» (ibidem, p. 18) consiste à refuser la double tentation de résister et de céder aux événements. Il ne s'agit ni de devenir " comme maitre et possesseur de la nature " ni de prendre, comme un caméléon, la couleur des circonstances : il faut devenir soi-même un «pur événement » et laisser les choses suivre leur cours. À cette condition, au lieu de se fracasser contre les faits ou de s'agripper à eux, comme un nageur plongé dans le cours furieux d'un torrent, on fraie sa course au milieu des événements en consentant à leur libre puissance. Comme le dit également Simmel : «La vie humaine présente un double aspect : celui de la causalité, du caractère simplement naturel de son cours - celui de la signification, qui en tant que sens, ouvrage et but, l'illumine ou l'inspire; ou, à un autre point de vue, nous tirons d'un côté notre mesure et notre ordre des mouvements de l'univers, tout en sentant et en conduisant d'un autre côté notre existence individuelle à partir d'un centre intime, en tant que responsabilité de soi et forme en quelque sorte fermée sur soi » (ibidem, p. 14).

10 Cette unité interne de l'individu, ou de l'agent historique, n'est à proprement parler ni un être ni une essence: Simmel ne reconstitue, en arrière-fond des sciences historiques, ni une ontologie ni une théologie. Il invoque pourtant le postulat de l'âme: " eine nie absolut zu bewahrheitende Vermutung », « une supposition destinée à rester à tout jamais sans confirmation absolue » (I.u.F., p. 62). Il veut dire par là que toute action, toute émotion et toute pensée portent la signature de leur auteur : l'existence des hommes, même quand elle est soumise à des pressions et à des violences, garde un caractère individuel et continu. Nous ne cessons jamais, même à notre insu, d'apposer une marque singulière sur ce que nous vivons, sur ce qui nous advient. Cette constatation ne destitue aucunement l'analyse extérieure et causale de son importance: les causes productrices et perturbatrices, les causes matérielles constituent bien l'étoffe réelle du monde, pèsent de leurs déterminations décisives sur les situations collectives et sur les destins individuels; toutefois, elles n'abolissent ni la forme ni la fin de ces mêmes destinées. Celui qui est charrié, malmené, endommagé par ces forces n'en porte pas moins en lui l'image et la forme de l'humanité tout entière, comme de sa singularité unique : l'art et la religion opposent leur pouvoir totalisant au pouvoir nu et dissociatif des forces. On retrouve en Simmel quelque chose du Montaigne qui, en pleine guerre civile, laisse ouvertes ses portes, remarquant : «Entre tant de maisons armées, moi seul, que je sache en France, de ma condition, ait fié au ciel la protection de la mienne » (Essais, II, 15). La non-action résolue devient principe d'action et renverse une situation.

11 "L'une des perceptions qui nous révèlent de la façon la moins ambiguë et la plus frappante l'âme des autres est leur regard. Or là, justement, nous n'avons aucun élément de comparaison avec nous-même» (I.u.F., p. 64). En revanche, nous ne saisissons à l'extérieur que ce dont nous portons en nous le moule ou l'idée: 
comprendre est une action qui dépend non seulement de l'entendement mais de l'âme, c'est-à-dire d'une aptitude esthétique, éthique et spirituelle à interpréter en termes de liberté, d'expérience et de destin des signes qui, quand ils nous parviennent du monde extérieur en tant qu'informations, n'ont encore qu'un statut indécis.

De cette dimension transhistorique de l'historique, Simmel multiplie les preuves: il montre, nous l'avons dit, que l'interprétation des œuvres d'art met au jour, légitimement, des sens que l'auteur n'a pas aperçus. Plus profondément, Simmel suggère que la compréhension de l'individuel, du singulier, de l'historique, ne peut jamais progresser que par variations : le désir naïf de coller au concret, de restituer le réel « tel qu'il s'est passé ", dérive d'une illusion. L'intelligible est d'abord un possible : l'art, la poésie, le roman ou le théâtre, en procédant de l'imagination et de l'esprit, proposent leurs codes, leurs hypothèses unifiantes, que nous comparons aux signes fragmentaires que constituent documents et témoignages. Le positivisme, le réalisme restent, en histoire, des idéologies impraticables. Pour qu'un événement ou un acteur prennent place dans la trame d'un récit, il faut les lester d'une continuité temporelle, d'une identité, d'une référence à une subjectivité unificatrice. C'est une nécessité aussi impérieuse pour l'historien que la démonstration pour le mathématicien. En établissant par touches, tout au long de ses essais et de ses livres, ces règles de constitution de l'histoire, Simmel ne renie pas Kant, il inaugure un nouveau style de philosophie sociale. De Kant, il conserve la vigilante attention aux formes selon lesquelles des objets et des relations sont donnés ou plutôt activement produits par notre esprit. L'histoire, à cet égard, s'inscrit dans le cadre de la philosophie transcendantale. Pourtant, la constitution des objets s'allie à l'interprétation des signes. En effet, ce qui figure dans l'histoire à titre de réalité est indissolublement événement inséré dans le tissu des causes et signification à rapporter à des agents qui opèrent à la manière de sources à partir desquelles une action commence. L'herméneutique n'est pas une discipline superflue: elle appartient à l'essence de l'histoire. L'acte de comprendre fait la synthèse de deux éléments initialement disjoints : "Des informations factuelles qui, en tant que telles, ne sont pas comprises, sont d'abord données. Sur cette base, sourd du sujet, à qui ces éléments apparaissent, soit spontanément soit au terme d'un travail, une pensée qui illumine et comprend ce donné » (I.u.F., p. 62).

Simmel voit bien que l'histoire est pleine de répétitions : aux actes qui inaugurent se mêlent les actes bien plus nombreux qui copient. Ils sont le signe de notre errance, de notre incapacité à mener jusqu'au bout notre Lebensintention, projet de vie, dont nous n'avons pas la claire conscience, mais que, de temps à autre, des signes nous font pressentir. Nous répétons, faute de décrire d'un seul mouvement inspiré notre trajectoire, notre visée.

14 Proche en cela de Rilke, Georg Simmel pense que la vertu éthique majeure, celle qui modèle les actions, et par là l'histoire, est la faculté si peu répandue de tenir debout sous le destin, de regarder la réalité comme elle est, au lieu de nous voiler les yeux, parce que nous en redoutons la grandeur, les énigmes ou l'horreur. On ne peut qu'être frappé par la similitude de leurs conceptions du destin. Nous avons évoqué celle de Simmel. Dans la huitième des Élégies de Duino, Rilke, après avoir opposé la créature qui "de tous ses yeux voit l'ouvert » à l'homme qu'à peine enfant déjà, «nous ployons à regarder en arrière, dans l'Apparence, et non pas dans l'Ouvert », définit ainsi le destin :

Dieses heisst Schicksal : gegenüber sein

und nichts als das und immer gegenüber 
[Ce qui s'appelle le destin, c'est cela : être en face, rien d'autre que cela et toujours être en face].

15 On peut qualifier une telle attitude, nous l'avons noté, à la fois de rationaliste et de mystique. Rationaliste, en ce qu'elle vise à regarder et à accepter le réel tel qu'il est, sans détourner de lui son regard. Mystique, car la capacité de consentir au réel n'est pas vue comme une simple ascèse intellectuelle.

Le réalisme est une vertu, mais, contrairement à ce qu'on croit, ce n'est pas une disposition naturelle; c'est tout au contraire une rupture avec l'attitude naturelle, une suspension des projections que sont nos représentations. Nous n'échappons pas à la représentation; nous pouvons tenter d'échapper aux pièges du réalisme naïf. Cette essai pour atteindre à l'impartialité et à la compréhension exige non seulement du savoir et du jugement, mais une manière d'être plus secrète, qu'on ne peut pas décrire, qu'on exhibe ou non. Sur cette vertu ultime, nécessaire à ceux qui, artistes, philosophes, ou historiens étudient l'homme, Simmel ne s'est pas étendu. A lui s'applique aussi la remarque trop souvent citée de Wittgenstein : «Wovon man nicht sprechen kann, darüber muss man schweigen » («ce dont on ne peut pas parler, il faut le taire »).

C'est pourtant à partir de ces convictions tacites, de ce secret aussi bien scellé que la tombe nue sous laquelle il repose dans le cimetière ouest de Strasbourg, que se sont organisées les entreprises scientifiques de Georg Simmel. Comme il voyait bien que du passé nous ne gardons que des fragments, on a cru à tort qu'il n'était capable que d'esquisses et de vues incomplètes. Comme il percevait en l'art l'une des voies majeures vers la vérité et l'intériorité des hommes, on l'a pris pour un esthète. Comme il avait évalué l'importance du phénomène religieux, on l'a taxé de spiritualisme. Comme il traitait l'action en aventure, on l'a trouvé romanesque. Il était seulement rigoureux et honnête.

18 Un peu à la façon de Helmholtz ou de Meyerson, Simmel ne croit pas qu'il y ait rupture entre connaissance ordinaire et connaissance scientifique. Comprendre, dans la vie de tous les jours et en histoire, est le même acte. La science est seulement plus consciente de ses opérations, de ce qu'elles ont d'hypothétique, de risqué ou de solide. La compréhension historique, notamment, est une modalité de l'intersubjectivité, de la relation humaine. Comme l'acte du médecin hippocratique, qui conjugue le diagnostic, le pronostic et le traitement, l'acte de l'historien combine la mise en évidence des situations, la restitution du cours des choses, et l'évaluation des causes productrices ou des projets qui provoquent des changements. On a nommé « relativisme » les scrupules rationalistes de Simmel: il se refuse à identifier un système de représentations, si cohérent ou informé soit-il, à la réalité elle-même. "En effet, note-t-il, combien de fois l'homme mûr se sent-il incapable de saisir ce qu'il a fait ou éprouvé comme jeune homme » (I.u.F., p. 65). Si notre propre passé se dérobe à nos prises, à plus forte raison les actions et les pensées des autres nous échappent-elles. Thucydide l'avait souligné, en reconstruisant les harangues des acteurs principaux de la Guerre du Péloponnèse : "Comme il m'a semblé que les orateurs devaient parler pour dire ce qui était le plus à propos, eu égard aux circonstances, je me suis efforcé de restituer le plus exactement possible la pensée complète des paroles exactement prononcées " (I, 22). L'histoire rationalise ; elle brosse un tableau qui comporte peut-être plus d'ordre que n'en ont eu les événements. Cette contrainte est interne et tient aux structures de l'acte même de 
comprendre, dans la vie et l'histoire. L'inscrutabilité du lien entre le récit historique et ce qui a eu lieu n'empêche pas d'affirmer l'existence d'une "vraie " version : comme Tarski, Simmel proclame son attachement au concept sémantique de vérité, quitte à ajouter que, dans les faits, la tâche dépasse nos prises effectives. Prenant l'exemple de la compréhension historique du Faust de Goethe, il déclare: «Eine Mehrheit von historischen, aus dem seelischen Vorgang geschöpften Verständnissen der Faustentstehung, die alle ebenso richtig wären, [...] ist ein Nonsens » (I.u.F., p. 73), c'està-dire : "Qu'il existe plusieurs manières de comprendre historiquement, en tant que création issue de l'âme, la naissance de Faust, et que toutes soient également vraies, est un pur non-sens »). Ce non-sens ontologique s'accompagne dans les faits d'une pluralité d'interprétations. Simmel n'est pas relativiste, il discerne les limites de l'effectuation d'un acte, dont le paradigme est clair, et les conditions de réalisation imparfaites.

De cette finitude intrinsèque de la compréhension historique, Simmel a tenté de tirer parti : puisque nous n'avons accès qu'à des épisodes fragmentaires de la vie des individus ou des groupes, il faut que le sociologue ou l'historien, tel l'artiste, choisisse l'événement révélateur, la crise ou l'incident le plus chargé de sens. À travers la partie, nous visons toujours une totalité. Entre des signes disjoints, nous cherchons « le pont et la porte », "Brücke und Tür ». L'art rend l'invisible visible et nous initie, à l'aide de quelques signes, à des existences qui, prises en elles- mêmes, forment des totalités. Simmel a inauguré en sociologie un nouveau style, non par esthétisme, mais par conviction métaphysique: il croyait, comme Racine ou Baudelaire, à la véracité de l'imagination constructive, à la nécessité, en sociologie, d'aborder indirectement le factuel, à l'aide de modèles théoriques, d'expériences de pensée. Là prend racine sa conviction, ultérieurement reprise par Max Weber, que tout savoir historique inclut dans sa trame des éléments transhistoriques, qui jouent le rôle de types idéaux. La constitution de l'histoire met en jeu des contenus "libres du temps", il y a dualité entre « seelischen und zeitfreiem Inhalt » («contenu inhérent à l'âme et étranger au temps ») (I.u.F., p. 76). Pour établir cette évidence, Simmel analyse ce que veut dire comprendre Kant: à côté de la restitution psychologique et singulière de son entreprise, il existe un autre mode d'intellection de son œuvre, indépendante de l'histoire et en même temps "sachlich». Il en va de même de toutes les autres productions humaines, scientifiques, techniques, et même politiques. Elles sont à la fois singulières et universelles, uniques et typiques, individuelles et génériques. L'art met en lumière l'élément universel. Plus généralement, la culture " est la voie de l'unité fermée à l'unité déployée, en passant par la multiplicité déployée » (Ph. Kultur, p. 185).

L'art tient de près à la religion, il comporte, quand il est grand, un élément sacré, une aspiration à l'Un. Simmel est à l'opposé de Dürkheim: il n'essaie pas de réduire le religieux à du social. Non par conviction personnelle, mais par méthode, par intuition des types idéaux et par-là des essences. Dans un comportement, individuel ou commun, il cherche à trier ce qui est balbutié et visé de ce qui est pratiquement réalisé : bien sûr, tout, en un sens, est socialement déterminé ; pourtant, dans tout ce que les hommes entreprennent, il y a une intention, qui n'est en dernier ressort intelligible que rapportée à une mesure ou à un patron, qui procède de l'âme, de l'homme tel qu'il serait si la société ne le pétrissait pas, n'altérait pas ses projets ultimes, sa forme interne. «Dieu n'est pas l'homme agrandi, mais l'homme est Dieu en miniature » (Ph. Kultur, p. 166). Les religions offrent des chemins et des signes, qui tous mènent l'homme sincère à une unique alternative: "harte Frage des Seins oder Nichtseins" («rue question de l'Être ou du Non-Être») (ibidem, p. 182). Dans cette prise en compte de la 
dimension éthique et spirituelle des destins individuels et du cours général de l'histoire, Simmel apparaît encore comme un initiateur, même s'il n'a pas à son actif des réalisations aussi importantes que celles de son ami Max Weber.

Il a écrit sur l'aventure et sur le salut de l'âme: cette rencontre n'est pas fortuite. L'action vise deux buts : transformer le monde extérieur et faire changer d'état aux hommes. Elle se déploie causalement dans le monde réel, et symboliquement dans l'être intime des agents. Outre le sens qu'elle a pour ceux qui agissent ou pâtissent, toute action propage ses effets loin de sa source. Simmel, qui fut lui-même plutôt un homme de rayonnement ou d'influence que d'action, avait l'intelligence de l'action : il ne caractérisait pas la scène où opèrent des acteurs par ses structures, par ce qu'un spectateur en apercevait; il y discernait des champs de forces, des opportunités ou des obstacles; il y lisait des signes interprétables. Il voyait que l'action, même quand elle exige de longues préparations, procède d'une crise et se joue dans l'instant, qui est indissolublement centre et partie, « Mittelpunkt » et « Teil » (Philosophische Kultur, p. 13, Das Abenteuer). Telle est la clef de son laconisme métaphysique.

L'action résout en pratique le paradoxe que soulève, dans l'ordre spéculatif, tout essai pour connaître l'homme: la partie y vaut pour le tout. "Qu'un événement isolé et contingent puisse receler une nécessité et un sens détermine le concept d'aventure ", repoussant par contraste à la périphérie le fil ordinaire de la vie (Ibidem, p.13-14). Certes, l'aventure est en marge; bien qu'elle constitue un tout, elle demeure une parenthèse. Elle est en même temps révélation et symptôme: "Nous sommes les aventuriers de la Terre" (Ph. Kultur, p. 26). Simmel souligne cet élément de rêve qui anime ou emporte les actions. L'aventure représente un signe des temps, du cours indécis et imprévisible de l'histoire.

En résumé, l'apparente dispersion de l'œuvre de Simmel s'explique par sa philosophie, par sa vision originale et rigoureuse des conditions très limitatives d'une connaissance de l'homme. Son anthropologie est cohérente, son rationalisme ancré, son sens des plans multiples, selon lesquels s'ordonne et se comprend le destin, aigu. Brassant, de ce fait, une matière riche et hétérogène, dont il sentait, presque avec angoisse, le caractère irrémédiablement lacunaire, Simmel a varié les approches et les genres. Il a usé de toutes les ressources d'un tempérament philosophe et artiste, pour demeurer fidèle à des exigences kantiennes d'ordre et de cohérence. Il n'a pas oublié que la raison est indissolublement un instrument de connaissance et un système de fins. De ces exigences conjuguées résulte le caractère attachant et souvent méconnu d'une œuvre qui, moderne dans sa forme, résiste au temps.

Il a été, pour Hannah Arendt, pour Walter Benjamin, ou déjà pour Max Weber, une référence et une source. Il possédait ce don mystérieux, indispensable au sociologue, à l'historien ou au politique, de découvrir, sur la base d'informations factuelles et limitées, le sens d'une situation, l'enjeu d'un choix, ou le caractère unique d'un être. Cette forme de pénétration devait être fascinante et irritante. De cet agacement, Raymond Aron laissait percer quelques signes : un savant jouait au magicien. En fait, Simmel tentait, au-delà des régularités et des généralités de la science ordinaire, d'atteindre à une connaissance du troisième genre, celle des réalités singulières. Il y employait avec l'insouciance d'un homme libre les ressources fondues de la sensibilité et de la raison. 


\section{NOTES}

1. - Das Individuum und die Freiheit Essais (1957), éd. Klaus Wagenbach Berlin, 1984.

Philosophische Kultur Gesammelte Essais (1923), éd. Klauss Wagenbach Berlin, 1983.

\section{RÉSUMÉS}

L'apparente dispersion de l'œuvre de Simmel s'explique par sa vision originale et rigoureuse des conditions d'une connaissance de l'homme. Il peint les plans multiples selon lesquels s'ordonne le destin des individus. Brassant une matière riche et hétérogène, dont il éprouve, presque avec angoisse, le caractère lacunaire, il varie les approches et les genres: il veut ainsi établir graduellement, tout au long de ses essais et de ses livres, les règles de constitution de l'histoire. L'idéal d'un Ranke, assignant à cette dernière de restituer «ce qui s'est réellement passé » lui semble inaccessible ; l'historien a pour tâche de mettre en relief, dans la profusion indéfinie des événements, des actions entremêlées qui tissent le cours intelligible des choses. Simmel ne renie pas Kant, il inaugure un nouveau style de philosophie sociale. En effet, ce qui figure dans l'histoire à titre de réalité, est indissolublement événement inséré dans un tissu de causes et signification à rapporter à des agents. L'herméneutique appartient à l'essence de l'histoire. Audelà des régularités et des généralités de la connaissance ordinaire, Simmel essaie d'atteindre à une connaissance rigoureuse des réalités singulières.

Das Werk Simmeis, so scheint es, hat einen ziemlich zerstreuten Charakter, und dies erklärt sich durch seine originale und strenge Auffassung der Bedingungen unter welchen die Kenntnis vom Menschen möglich ist. Er beschreibt die verschiedenen Ebenen nach welchen sich das Schicksal des Einzelmenschen abzeichnet. Da er mit einem verzweigten und vielfältigen Material arbeitet, dessen Unvollständigkeit und Mängel er sehr stark empfindet, geht er von verschiedenen Standpunkten aus, und nach verschiedenen Verfahren vor: er will so in seinen Schriften und Essais, von einem Werk zum anderen, stufenweise die Regeln der Geschichtsforschung aufstellen. Das Ideal eines Ranke, welcher der Geschichte die Aufgabe zuschreibt, «das was wirklich geschah» wiederzugeben, scheint ihm unerreichbar; der Geschichtsforscher bezweckt, unter den unbeschreibbar vielfältigen Ereignissen, die sich darbieten, miteinander verflochtene Fakten und Taten ins Licht zu rücken, welche den begreifbaren Verlauf der Dinge abmachen.

Es geht Simmel nicht darum, Kant zu verleugnen, er beginnt bloss auf eine neue Art Gesellschaftsphilosophie zu treiben. Denn das, was die Geschichte als geschehene Tatsache betrachtet, ist notwendigerweise mit einem Kausalzusammenhang verbunden und Träger einer Bedeutung. Hermeneutik gehört zum Wesen der Geschichte. Über die Allgemeingültigkeiten der gewöhnlichen Erkenntnis hinausgehend, versucht Simmel, eine strenge Erkenntnis der Einzelfakten anzustreben. 